\title{
Eröffnungsansprache zum Vierten Meeresbiologischen Symposion, Hamburg, 1963
}

\author{
OtTo Kinne \\ Biologische Anstalt Helgoland, Zentrale, Hamburg-Altona
}

Meine sehr verehrten Damen und Herren!

Es ist mir eine große Ehre, Sie hier in Hamburg auf das herzlichste willkommen zu heißen. Ich danke Ihnen, daß Sie in so großer Zahl der Einladung zur Teilnahme am Vierten Meeresbiologischen Symposion Folge geleistet haben. Insbesondere freue ich mich über das rege Interesse, welches dieses Symposion auch im nahen und fernen Ausland gefunden hat. Die Anwesenheit namhafter Wissenschaftler aus den europäischen Nachbarstaaten und aus Nordamerika sowie die zahlreichen Briefe und Telegramme, welche dieser Veranstaltung Grußbotschaften und gute Wünsche übermitteln, sind ein beredter Beweis für dieses Interesse und verleihen dem Symposion besondere Bedeutung.

Mein herzlicher Dank gilt all denen, die sich als Vorsitzende oder Vortragende sozusagen als "Hauptdarsteller" - in den Dienst der guten Sache gestellt haben. Er gilt jenen wissenschaftlichen, administrativen und technischen Mitarbeitern, deren emsige Tätigkeit sich unter der Oberfläche des allen sichtbaren Tagungsablaufes abspielt. Er gilt den Kollegen von der Bundesforschungsanstalt für Fischerei, insbesondere den Herren Professoren von Brandt und Meyer-Waarden, die uns bei der Vorbereitung des Symposions nach Kräften unterstützten. Beide Kollegen entbieten den Symposions-Teilnehmern freundliche Grüße. Sie sind zur Zeit in Bremerhaven, wo heute das gerade fertiggestellte Forschungsschiff „Walther Herwig“ in Dienst gestellt wird und werden daher erst später zu uns kommen. Nicht zuletzt gilt unser Dank dem Herrn Bundesminister für Ernährung, Landwirtschaft und Forsten und seinem Ministerium für die entgegenkommende Unterstützung bei der Vorbereitung und Durchführung dieser Veranstaltung.

Die Idee zur alljährlichen Abhaltung eines Meeresbiologischen Symposions geht auf die Zeit unmittelbar nach der Wiedereröffnung der Meeresstation der Biologischen Anstalt Helgoland in Jahre 1959 zurück. Sie entsprang dem Wunsche, die modernen und großzügigen Einrichtungen der Meeresstation auf Helgoland mit neuem, pulsierendem wissenschaftlichen Leben zu erfüllen. Mein Vorgänger, Professor A. BückMANN - der sich um den Wiederaufbau der Biologischen Anstalt Helgoland große Verdienste erworben hat - lud 1960 zum Ersten Meeresbiologiscben Symposion nach Helgoland ein. Inzwischen ist die ursprüngliche Konzeption eines alljährlichen „Helgoländer Symposions" erweitert worden. 1961 fand das Zweite Meeresbiologische Sym- 
posion im Institut für Meereskunde in Kiel statt und 1962 das Dritte im Institut für Meeresforschung in Bremerhaven. Die Erfolge, welche diesen Veranstaltungen beschieden waren, haben zu dem allgemeinen Wunsch geführt, die Reihe der jährlichen Meeresbiologischen Symposien möge zu einer Dauereinrichtung werden - zu einer permanenten Plattform für den Gedanken- und Erfahrungsaustausch von Meeresbiologen. Die Biologische Anstalt Helgoland beabsichtigt auch in Zukunf, Arbeit und Frïchte, welche mit der Durchführung derartiger Veranstaltungen verbunden zu sein pflegen, mit anderen meeresbiologischen Institutionen zu teilen. Sie wird in jedem dritten Jahr die Rolle des Veranstalters übernehmen.

Im Gegensatz zu den vorangegangenen drei Meeresbiologischen Symposien, welche nationalen Charakter trugen, ist das vierte Symposion eine internationale Veranstaltung. Meine Kollegen von der Biologischen Anstalt Helgoland und ich begrüßen diese Entwicklung. Problematik und Aufgabenbereich der modernen Meeresbiologie stellen heute Anforderungen, welche die personelle und finanzielle Kapazität einzelner Nationen weit übersteigen. Meeresbiologie ist zu einem internationalen Anliegen geworden, zu einem der vordringlichsten und umfangreichsten Forschungsvorhaben des zwanzigsten Jahrhunderts. Es fehlt nicht an Stimmen, die der Meeresforschung eine größere unmittelbare Bedeutung beimessen als der Weltraumforschung. Amerikanische Politiker haben dem geflügelten Begriff „Atomzeitalter" den neuen Begriff vom "Meereszeitalter" an die Seite gestellt. Über 70\% der Erdoberfläche wird vom Meer bedeckt. Progressiv zunehmende Bevölkerungszahlen, bedrohliche Zukunftsperspektiven der Nahrungsversorgung, die Bedeutung des Meeres als Rohstoffquelle und als Verkehrsweg sowie das zunehmende Sicherheitsbedürfnis der politischen Mächtegruppen repräsentieren - im Verein mit dem wachsenden wissenschaftlichen, technischen und apparativen Potential der Kulturnationen - eine Konstellation von Gegebenheiten, welche mit elementarer Kraft einen Wettlauf um das Wissen vom Meer ausgelöst hat.

Was wissen wir über das Leben im Meer? Erschreckend wenig! Gewiß: es sind einige Dutzend Expeditionen durchgeführt worden. Gewiß: die meisten dieser Expeditionen verliefen außerordentlich erfolgreich und erbrachten eine Fülle neuer Informationen, etwa über bisher unbekannte Tier- und Pflanzenarten, über die Verbreitung und Abundanz wichtiger "Schlüssel-Arten" oder über das Leben in großen und größten Tiefen. Ebenso gewiß hat die Fischereiforschung in jahrzehntelanger Arbeit wichtige Erkenntnisse über die Verbreitung von Nutzfischarten, über Fischwanderungen, Laichplätze und über die Biologie einiger Nahrungstiere der Fische gesammelt. Aber recht eigentlich war eine meeresbiologische Forschung bisher nur innerhalb sehr eng gesteckter Grenzen möglich. Es mangelte an ozeantüchtigen Forschungsschiffen, an Personal und Mitteln. So stehen wir denn am Anfang: Ozeanweite Meeresbiologie, wie sie uns als eine große, verpflichtende, internationale Aufgabe vorschwebt, ist eine junge Forschungsdisziplin. Sie ist Meilenstein zugleich und Wendepunkt auf dem langen Wege einer fruchtbaren Entwicklung, welche vor etwa 80 Jahren begann und in der Problematik der klassischen Zoologie und Botanik wurzelte. Damals galt es, die faszinierenden Konzeptionen Darwins zu prüfen und auszubauen. Die Fauna und Flora der marinen Küstenbereiche lieferte dabei ein reichhaltiges und lohnendes Untersuchungsmaterial. Prominente Wissenschaftler der Universitäten und anderer inländischer Forschungseinrichtungen wurden in großer Zahl angezogen von den neu errichteten Mee- 
resstationen an den Küsten Europas und Nordamerikas und leisteten hier hervorragende Arbeit. Sie waren es, die hier den Geist und die wissenschaftliche Atmosphäre prägten. Später widmeten sich die Gastforscher dann vor allem entwicklungsmorphologischen und physiologischen Studien. Dieser Zeit verdankt die Biologie viele vortreffliche, grundlegende Untersuchungen und neue Impulse.

Heute besitzen viele Forschungsinstitute des Inlandes eigene Seewassersysteme, in denen sich vielerlei Untersuchungen an marinen Organismen, auch in weiter Entfernung vom Meer, durchführen lassen. Große und wichtige Zweige der modernen Biologie - wie etwa die Biochemie und die Molekularbiologie - arbeiten vornehmlich an „Laboratoriumstieren". Die für derartige Arbeiten benötigten komplizierten Apparaturen lassen sich nicht oder nur mit Schwierigkeiten transportieren. So hat sich die eigentliche Gastforschertätigkeit immer mehr auf ökologische, physiologische und ethologische Untersuchungen konzentriert, für welche die Arbeit direkt am oder im Meer obligatorisch ist. Im Verein mit der Intensivierung der Meeresforschung hat diese Entwicklung in jüngster Zeit dazu geführt, daß die eigene wissenschaftliche Arbeit an den meeresbiologischen Institutionen mehr und mehr an Bedeutung gewonnen hat. So besitzen heute an vielen marinbiologischen Einrichtungen Gastforschung und Eigenforschung gleichen Rang. Wer heute am Meer arbeiten will, sucht sich "seine" Meeresstation vor allem nach drei Gesichtspunkten aus: der ökologischen Situation, den Arbeitsbedingungen und - nicht zuletzt - der wissenschaftichen Konzeption und Bedeutung der Station.

In der Biologischen Anstalt Helgoland sind wir uns dieser Entwicklung bewußt: In zahlreichen Besprechungen und Diskussionen haben wir unsere Forschungsziele und wissenschaftlichen Konzeptionen erörtert und neu orientiert. Es würde zu weit führen, wollte ich darauf hier im einzelnen eingehen. Ich möchte Sie aber in aller Kürze mit den wichtigsten Punkten unseres neuen, bereits angelaufenen Forschungsprogramms bekanntmachen. Nach unserer Auffassung repräsentiert die detaillierte, langfristige und konsequente Erforschung der ökologischen Dynamik im Meer, verbunden mit einer umfassenden quantitativen und qualitativen Analyse der Aufbau-, Umbau- und Abbauvorgänge, das vordringlichste meeresbiologische Forschungsvorhaben der Gegenwart. Ein solches Vorhaben gehört gleichzeitig aber auch zu den schwierigsten und komplexesten wissenschaftlichen Unternehmungen. Es erfordert eine synoptische oder doch quasi-synoptische Erfassung zahlreicher biologischer, chemischer und physikalischer Parameter. Diese Parameter müssen in regelmäßigen Zeitintervallen an zahlreichen Stationen und in verschiedenen Wassertiefen gemessen werden. Das führt zu einer gewaltigen Anzahl von Proben, welche bearbeitet, ausgewertet und interpretiert werden müssen. Hier ist eine Umstellung auf rationellere Methoden unumgänglich. In zunehmendem Maße müssen kontinuierliche Meßreihen an die Stelle von Einzelmessungen treten und langwierige Messungen oder Zählungen von Hand durch automatische Registriergeräte ersetzt oder doch ergänzt werden.

Selbst bei Beschränkung auf ein sehr kleines Meeresgebiet setzt die Durchführung eines derartigen Forschungsprogramms eine enge Zusammenarbeit innerhalb einer Gruppe von Wissenschaftlern und Technikern voraus und stellt an alle Mitglieder einer solchen Arbeitsgruppe hohe fachliche und physische Anforderungen. Größere Ozeangebiete können mit ausreichender Aussicht auf Erfolg nur durch mehrere solcher Ar- 
beitsgruppen und im Rahmen einer großzügigen internationalen Zusammenarbeit erforscht werden.

Unser Programm einer eingehenden Analyse des Gesamtstoffwechsels geeigneter Meeresgebiete fußt zu etwa gleichen Teilen auf Untersuchungen im Meer und im Laboratorium. Diese „kombinierte Methode“ gilt als sicherstes Mittel, Fehlinterpretationen von ausschließlich im Meer oder ausschließlich im Laboratorium erarbeiteten Ergebnissen zu vermeiden oder doch zu verringern.

Bei den Untersuchungen im Meer unterscheiden wir: a) Terminfahrten im Gebiet der Nordsee und b) Teilnahme an nationalen und internationalen Gemeinschaftsunternehmungen in weiter entfernten Meeresgebieten. Unsere Terminfahrten in der Nordsee sollen sozusagen "vor der Haustür" mit eigener, auf den Habitat sowohl als auf die Struktur unserer Anstalt zugeschnittener Fragestellung durchgeführt werden. Das ermöglicht eine größere Spezifität der Fragestellung und eine größere Intensität und Kontinuität der Informationsbeschaffung, als das etwa bei internationalen Gemeinschaftsunternehmungen der Fall sein kann. Hier überlappen sich ja gelegentlich verschiedene Konzeptionen und Interessensphären, und demgemäß fordert die Ausarbeitung und Verwirklichung des Arbeitsprogramms infolge der vielseitigen Ansprüche an Raum, Zeit und Geräten vielfach Kompromisse, welche dem einen oder anderen Teilvorhaben abträglich sein können. Auf der anderen Seite ist die Teilnahme an internationalen Gemeinschaftsunternehmungen dringend erwünscht und für unsere Arbeiten eine Conditio sine qua non. So läßt sich beispielsweise der synoptische Einsatz mehrerer großer Forschungsschiffe und der mit einer weiträumigen Planung verbundene Großeinsatz von Personal und Apparaten finanziell nur durch internationale Zusammenarbeit realisieren. Zudem sind wir heute mehr als je zuvor auf einen ständigen Erfahrungsaustausch und engste Kontakte mit unseren ausländischen Kollegen angewiesen.

Bei den eigenen Untersuchungen im Gebiet der Nordsee kommt es vor allem darauf an, Mittel und Wege zu finden, um gleichzeitig eine größere Anzahl von biologischen, chemischen und physikalischen Informationen zu erhalten. Meßreihen mit wenigen Komponenten werden von zahlreichen Meeresstationen seit Jahren durchgeführt, und zwar mit relativ großem Arbeitsaufwand aber geringem Erfolg. Wenn wir uns vorteilhaft von diesem alten, unzureichenden Verfahren unterscheiden wollen, müssen wir die Anzahl der ständig registrierten Komponenten erheblich erhöhen. Dies um so mehr, als das mit unseren gegenwärtigen Mitteln erreichbare Untersuchungsgebiet durch hydrographische Inhomogenität charakterisiert ist. In der Anfangsphase unseres Nordseeprogramms werden seit Mai 1963 in regelmäßigen Intervallen drei Schnitte gefahren: Schnitt Helgoland - Feuerschiff P 8, Schnitt Helgoland - Elbmündung und Schnitt Helgoland - Mittelhever.

Die Untersuchungen im Laboratorium werden sich vor allem auf folgende Punkte konzentrieren:

1. Ausarbeitung einer geeigneten Zuchtmethodik. Nur an normalreagierenden, gesunden Organismen und unter adäquaten Umweltbedingungen gewonnene Ergebnisse sind zur Interpretation der ökologischen Situation in der freien Natur geeignet. 2. Zuchtversuche an Meeresorganismen in Aquarien und Teichkulturen. 3. Studium der Biologie (im weitesten Sinne) und der Lebensgewohnheiten der ökologisch wichtigsten Arten. 4. Untersuchungen über die Intensität von Wachstum, Stoffwechsel und Vermehrung 
unter verschiedenen Umweltbedingungen. 5. Ermittlung der gesamten funktionellen und strukturellen Reaktionsamplitude unter verschiedenen Umweltbedingungen sowie der Regulations- und Adaptationskapazität. 6. Pionierversuche über die Möglichkeit, die Hauptkomponenten des Okosystems (Primärproduzenten, Konsumer, Remineralisatoren) unter kontrollierten Laboratoriumsbedingungen in einzelnen, aber durch Röhren verbundenen Behältern „aufzubauen". Studium der Chemie und Physiologie eines vereinfachten "Mikro-Okosystems" im Laboratorium. 7. Analyse der Gesetzmäßigkeiten, welche die intra- und interspezifische Koexistenz bestimmen. Untersuchungen über die Bedeutung von Stoffwechselendprodukten als ökologische Wirkstoffe.

Zuchtversuche an Meerestieren sind bisher nur in geringer Zahl und oftmals ohne Erfolg durchgeführt worden. Das hat vielfach zu einer nachhaltigen Skepsis Anlaß gegeben, welche dann ihrerseits die gerade auf diesem Gebiet so notwendige Initiative gelähmt hat. Hier bedarf es eines frischen, energischen Neubeginns. Dem gewissenhaften, mutigen Pionier wird sich ganz sicher ein weites, lohnendes, neues Forschungsfeld eröffnen.

Neben diesem neuen Arbeitsprogramm werden die verschiedenen Forschungsrichtungen und Einzelvorhaben innerhalb der sieben Fachabteilungen unserer Anstalt beibehalten werden.

Zur Durchführung ihrer Forschungs- und Stationsaufgaben verfügt die Biologische Anstalt Helgoland über einen Stab von insgesamt 17 Wissenschaflern und 53 technischen und administrativen Angestellten sowie über einen seegehenden Forschungskutter, die „Uthörn“, und mehrere kleinere Boote für den Stationsbetrieb auf Helgoland und Sylt.

Der wissenschaftliche Stab gliedert sich in folgende Abteilungen: Zoologie, Botanik, Physiologie, Mikrobiologie, Planktonforschung, Ichthyologie und Strahlenbiologie. Die Einrichtungen der Anstalt befinden sich an drei verschiedenen Orten: die Zentrale in Hamburg, die Meeresstation auf der Insel Helgoland und die Litoralstation auf der Insel Sylt. Die Zentrale dient vor allem der unmittelbaren Zusammenarbeit und dem Gedankenaustausch mit fachverwandten Institutionen auf dem Festland, insbesondere mit der Meeres- und Fischereiforschung und den Universitäten. Neben Laboratorien, Kulturräumen, Arbeitsräumen und einer Bibliothek beherbergt sie die Direktion und Verwaltung der Anstalt. Die Meeresstation auf Helgoland - das Herz der Anstalt - zählt zu den größten und modernsten meeresbiologischen Forschungsstationen der westlichen Welt. Neben zahlreichen Laboratorien, Arbeitstäumen und vielerlei Spezialeinrichtungen für den eigenen wissenschaftichen Stab enthält sie 15 Gastforscherlaboratorien und zwei Kurssäle. Für die Gastforschung wurde zudem seitens der Deutschen Forschungsgemeinschaft eine Vielzahl moderner Apparaturen und Geräte zur Verfügung gestellt, so daß heute Forschungsarbeiten auf fast allen Gebieten der Biologie durchgeführt werden können. Die Anstalt verfügt auf Helgoland ferner über ein Züchtungslaboratorium am Südhafen, ein Gästehaus mit 25 Zimmern auf dem Oberland sowie über 9 Wohnhäuser für Anstaltsangehörige. Die Litoralstation in List auf Sylt ist in einem umgebauten Hafengebäude untergebracht. Sie dient vor allem der Erforschung litoraler Lebensräume, insbesondere der 
für den Nordseeraum so charakteristischen Watten, und bietet insgesamt drei Gastforschern eine Arbeitsmöglichkeit.

Eine auch noch so kurze Skizze über die Aufgaben der Biologischen Anstalt Helgoland wäre unvollständig ohne die Erwähnung unserer Lehraufgaben. An der Meeresstation auf Helgoland und an der Litoralstation in List auf Sylt halten Universitätsdozenten alljährlich meeresbiologische Kurse ab und führen zoologische und botanische Exkursionen durch. Gleichzeitig veranstalten wir in eigener Regie Kurse und Exkursionen für Studenten und Lehrer an höheren Schulen. Es steht zu hoffen, daß für die Betreuung und vor allem die Unterbringung der Kurs- und Exkursionsteilnehmer in Bälde angemessene Voraussetzungen geschaffen werden. Von besonderer Dringlichkeit ist in diesem Zusammenhang die Errichtung des seit langem geplanten Studentenheims. In jedem Jahr verschickt unser Versanddienst von Helgoland und List aus eine Vielzahl von Tier-, Pflanzen- und Seewassersendungen an festländische Forschungsinstitute, und über 100000 Besucher erfreuen sich in jedem Sommer an der lebendigen Formenmannigfaltigkeit marinen Lebens in unserem Seewasseraquarium auf Helgoland.

Meine Damen und Herren! Mit dieser kurzen Darstellung über Forschungspläne, Stab, Einrichtungen und Aufgaben der Biologischen Anstalt Helgoland möchte ich meine Ausführungen schließen. Ich bin überzeugt davon, daß die Vorträge und Diskussionen dieser internationalen Veranstaltung von großer Bedeutung sein werden für die Beurteilung des Einflusses der Veränderungen abiotischer Umweltfaktoren auf die Lebensvorgänge im Meer. Dem Symposion wünsche ich von ganzem Herzen einen harmonischen Verlauf und jedem von Ihnen reiche und fruchtbare Stunden des Gebens und Nehmens.

In diesem Sinne eröffne ich das Vierte Meeresbiologische Symposion 1963. 